\title{
The Order of Euler's Totient Function
}

\author{
Darrell Cox ${ }^{1}$, Sourangshu Ghosh ${ }^{2}$ and Eldar Sultanow ${ }^{3}$ \\ ${ }^{1}$ Department of Mathematics, Grayson County College, United \\ States \\ ${ }^{2}$ Department of Civil Engineering,Indian Institute of Technology \\ Kharagpur, India \\ ${ }^{3}$ Potsdam University, Chair of Business Informatics, Processes and \\ Systems, Potsdam, Germany
}

\begin{abstract}
The Möbius function is commonly used to define Euler's totient function and the Mangoldt function. Similarly, the summatory Möbius function (the Mertens function) is used to define the summatory totient function and the summatory Mangoldt function (the second Chebyshev function). Analogues of the product formula for the totient function are introduced. An analogue of the summatory totient function with many additive properties is introduced.
\end{abstract}

\section{Introduction}

If $n \geq 1$ the Euler totient function $\varphi(n)$ is defined to be the number of positive integers not exceeding $n$ which are relatively prime to $n$. If $n>1$, then $n=p_{1}^{a_{1}} p_{2}^{a_{2}} \cdots p_{k}^{a_{k}}$ where $p_{1}, p_{2}, \ldots, p_{k}$ are primes. The Möbius function $\mu(n)$ is defined as follows: $\mu(1)=1, \mu(n)=(-1)^{k}$ if $a_{1}=a_{2}=\cdots=a_{k}=1$, or $\mu(n)=0$ otherwise. Note that $\mu(n)=0$ if and only if $n$ has a square factor $>1$. The Euler totient function is related to the Möbius function through the following formula:

(1) $\varphi(n)=\sum_{d \mid n} \mu(d) \frac{n}{d}$

See Theorem 2.3 of Apostol's [1] book. The Mangoldt function $\Lambda(n)$ is defined to be $\log (p)$ if $n=p^{m}$ for some prime $p$ and some $m \geq 1$, or 0 otherwise. $\Lambda(n)$ is expressed in terms of the logarithm as follows:

(2) $\Lambda(n)=\sum_{d \mid n} \mu(d) \log \frac{n}{d}=-\sum_{d \mid n} \mu(d) \log (d)$

See Theorem 2.11 of Apostol's book. The second Chebyshev function $\psi(x)$ is defined to be $\sum_{n \leq x} \Lambda(n)$. The average order of $\varphi(n)$ is: 
(3) $\sum_{n \leq x} \varphi(n)=\frac{3}{\pi^{2}} x^{2}+O(x \log x)$ so the average order of $\varphi(n)$ is $3 n / \pi^{2}$.

See Theorem 3.7 of Apostol's book. A function used to prove $\lim _{x \rightarrow \infty} \frac{M(x)}{x}=0$ where $M(x)$ is the Mertens function is also generalized. This definition is:

(4) If $x \geq 1$ we define $H(x)=\sum_{n \leq x} \mu(n) \log n$.

See pg. 91 of Apostol's book. The definition of derivatives of arithmetical functions is:

(5) For any arithmetical function $f$ we define its derivative $f^{\prime}$ to be the arithmetical function given by the equation $f^{\prime}(n)=f(n) \log n$ for $n \geq 1$.

See section 2.18 of Apostol's book. The derivative concept can be used to derive a formula of Selberg which can be used as the starting point of an elementary proof of the prime number theorem. This formula is:

(6) The Selberg identity. For $n \geq 1$ we have $\Lambda(n) \log n+\sum_{d \mid n} \Lambda(d) \Lambda\left(\frac{n}{d}\right)=$ $\sum_{d \mid n} \mu(d) \log ^{2} \frac{n}{d}$.

See Theorem 2.27 of Apostol's book. This identity will also be generalized. Let $\Phi(x)$ denote $\sum_{n=1}^{x} \varphi(n)$. Let $M(x)$ denote $\sum_{n=1}^{x} \mu(n)$.

\section{The Redheffer Matrix}

The squarefree divisors of $n=2,3,4,5,6, \ldots$ are $2,3,2,5,(2,3,6), 7,2,3$, $(2,5,10), 11,(2,3,6), \ldots$. For example, for $n \leq 12$ there are $\lfloor 12 / 2\rfloor 2$ 's, $\lfloor 12 / 3\rfloor$

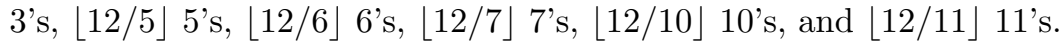

Mikolás [2] proved that $\sum_{i=1}^{x} M(\lfloor x / i\rfloor)=1$. In general, $\sum_{i=1}^{x} M(\lfloor x /(i n)\rfloor)=1$, $n=1,2,3, \ldots, x$ (since $\lfloor\lfloor x / n\rfloor / i\rfloor=\lfloor x /(i n)\rfloor)$. Let $R_{x}^{\prime}$ denote a square matrix where element $(i, j)$ equals 1 if $j$ divides $i$ or 0 otherwise. (In a Redheffer matrix, element $(i, j)$ equals 1 if $i$ divides $j$ or if $j=1$. Redheffer [3] proved that the determinant of such a $x$ by $x$ matrix equals $M(x)$.) Let $T_{x}$ denote the matrix obtained from $R_{x}^{\prime}$ by element-by-element multiplication of the columns by $M(\lfloor x / 1\rfloor), M(\lfloor x / 2\rfloor), M(\lfloor x / 3\rfloor), \ldots, M(\lfloor x / x\rfloor)$. For example, the $T_{x}$ matrix for $x=12$ is 


$\begin{array}{rrrrrrrrrrrr}-2 & 0 & 0 & 0 & 0 & 0 & 0 & 0 & 0 & 0 & 0 & 0 \\ -1 & -1 & 0 & 0 & 0 & 0 & 0 & 0 & 0 & 0 & 0 & 0 \\ -1 & 0 & -1 & 0 & 0 & 0 & 0 & 0 & 0 & 0 & 0 & 0 \\ -1 & -1 & 0 & -1 & 0 & 0 & 0 & 0 & 0 & 0 & 0 & 0 \\ 0 & 0 & 0 & 0 & 0 & 0 & 0 & 0 & 0 & 0 & 0 & 0 \\ 0 & 0 & 0 & 0 & 0 & 0 & 0 & 0 & 0 & 0 & 0 & 0 \\ 1 & 0 & 0 & 0 & 0 & 0 & 1 & 0 & 0 & 0 & 0 & 0 \\ 1 & 1 & 0 & 1 & 0 & 0 & 0 & 1 & 0 & 0 & 0 & 0 \\ 1 & 0 & 1 & 0 & 0 & 0 & 0 & 0 & 1 & 0 & 0 & 0 \\ 1 & 1 & 0 & 0 & 1 & 0 & 0 & 0 & 0 & 1 & 0 & 0 \\ 1 & 0 & 0 & 0 & 0 & 0 & 0 & 0 & 0 & 0 & 1 & 0 \\ 1 & 1 & 1 & 1 & 0 & 1 & 0 & 0 & 0 & 0 & 0 & 1\end{array}$

Let $U_{x}$ denote the matrix obtained from $T_{x}$ by element-by-element multiplication of the columns by $\varphi(j)$. The sum of the sums of the columns of $U_{x}$ then equals $\Phi(x) . i=\sum_{d \mid i} \varphi(d)$, so $\sum_{i=1}^{x} M(\lfloor x / i\rfloor) i$ (the sum of the sums of the rows of $U_{x}$ ) equals $\Phi(x)$. This relationship was proved by Cox [4] (and previously by Mikolás).

(7) $\sum_{i=1}^{x} M(\lfloor x / i\rfloor) i=\Phi(x)$

A plot of the sums of the rows for $x=8000$ is given in Figure 1 .

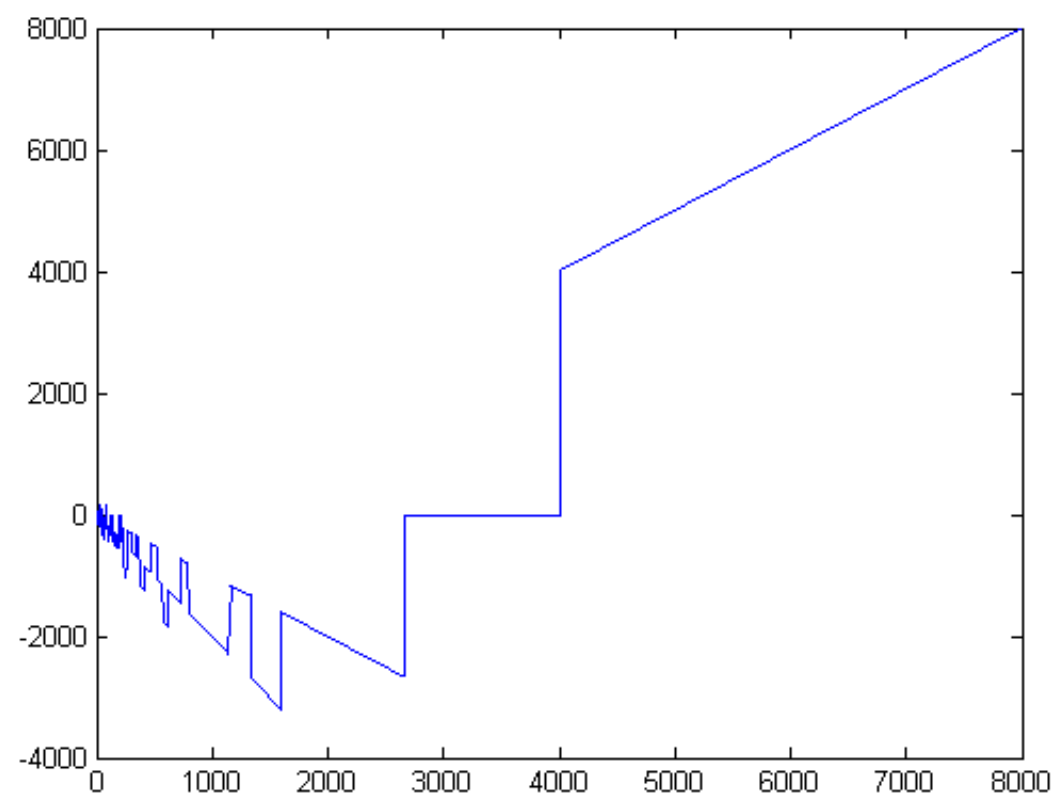

Figure 1: Plot of the sums of the rows for $x=8000$ 
This plot indicates that $\Phi(x)$ equals a series involving the squarefree numbers $1,2,3,5,6,7,10, \ldots$. For $x=200$, the values from $\lfloor x / 2\rfloor+1$ to $\lfloor x / 1\rfloor$ are 101 , $102,103, \ldots, 200$ (the initial value is $\lfloor x / 2\rfloor+1$ ). The values from $\lfloor x / 3\rfloor+1$ to $\lfloor x / 2\rfloor$ are 0 . The values from $\lfloor x / 5\rfloor+1$ to $\lfloor x / 3\rfloor$ are $-41,-42,-43, \ldots-66$. The values from $\lfloor x / 6\rfloor+1$ to $\lfloor x / 5\rfloor$ are $-68,-70,-80, \ldots-66$ (the initial value is the negative of twice $\lfloor x / 6\rfloor+1)$. The process continues with larger squarefree divisors. Several squarefree divisors may map to the same term in the series the smallest is selected. There are other multiples of the initial values and other multiples of 0 can occur. For $x=200$, the squarefree numbers and corresponding multiples are $(2,1),(3,0),(5,-1),(6,-2),(7,-1),(10,-2),(11,-1)$, $(13,-2),(14,-3),(15,-2),(17,-1),(19,-2),(21,-3),(23,-1),(26,-2)$, $(29,-1),(34,-3),(41,0),(51,-3),(67,-1),(101,1)$, and $(201,-8)$. The multiple for a squarefree number $q$ is $M(q-1)$. Each term in the series is the sum of an arithmetic progression and is thus quadratic in terms of $x$. About 0.81 times the first term equals $\Phi(x)$.

If $q<\sqrt{x},\lfloor x / q\rfloor \neq\lfloor x / r\rfloor$ where $r$ is the next squarefree number. The asymptotic density of the squarefree numbers is $\frac{6}{\pi^{2}}$. (Even for $x$ as small as 10 , this gives 6.08. The actual number is 6 . For $x<50$, this gives 30.40. The actual number is 30 .) The sum of the terms corresponding to the first $\left\lceil\sqrt{\frac{6 x}{\pi^{2}}}\right\rceil$ squarefree numbers is an approximation of $\Phi(x)$ typically accurate to less than $0.5 \%$ relative error for fairly large $x$ values (say greater than 300). A plot of $\Phi(x)$ and this approximation for $x=40,41,42, \ldots, 110$ is given in Figure 2. 


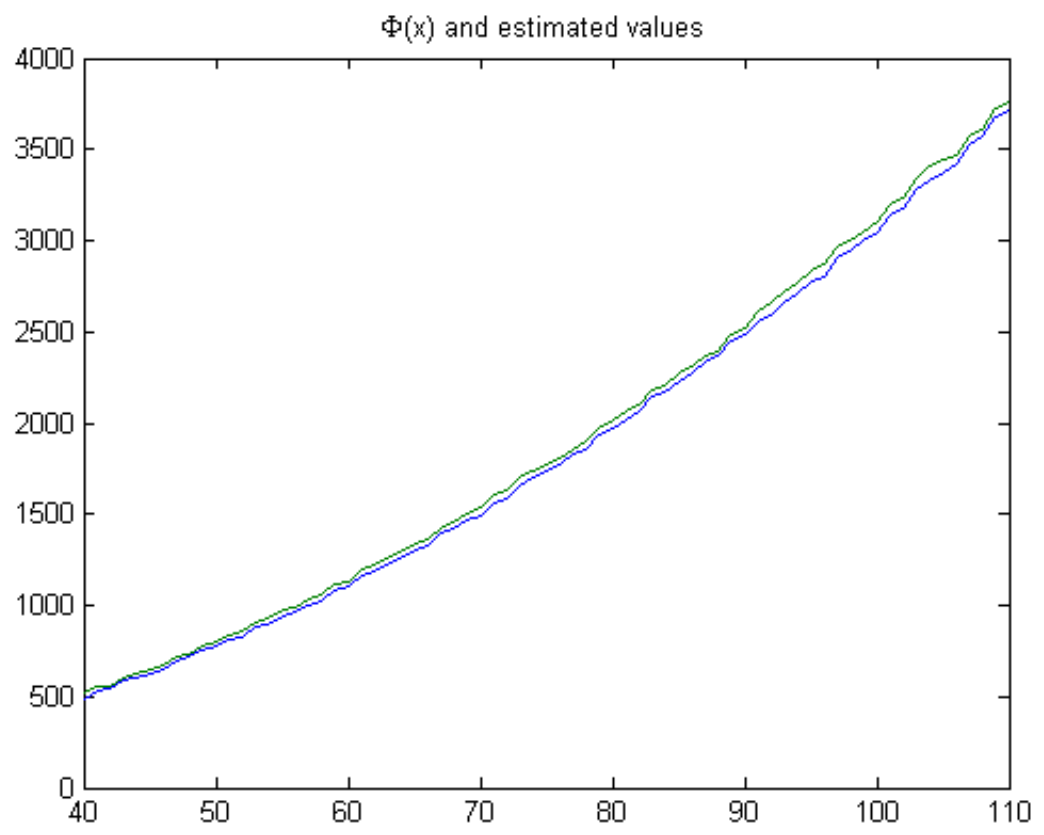

Figure 2: Plot of $\Phi(x)$ and this approximation for $x=40,41,42, \ldots, 110$

For $x=40$ to 41 , the squarefree numbers used are 2, 3, 5, 6, and 7. For $x=42$ to 59 , the squarefree numbers used are $2,3,5,6,7$, and 10 . For $x=60$ to 80 , the squarefree numbers used are $2,3,5,6,7,10$, and 11 (with 7 exceptions). For $x=81$ to 105 , the squarefree numbers used are $2,3,5,6,7,10,11$, and 13 (with 3 exceptions). For $x=106$ to 110, the squarefree numbers used are 2, 3, $5,6,7,10,11,13$, and 14 . For the first exceptions, there are no 11's so 13 's are used. For the second exceptions there are no 11's or 14's so 13's and 15's are used. A plot of the $\Phi(x)$ values is given in Figure 3. 


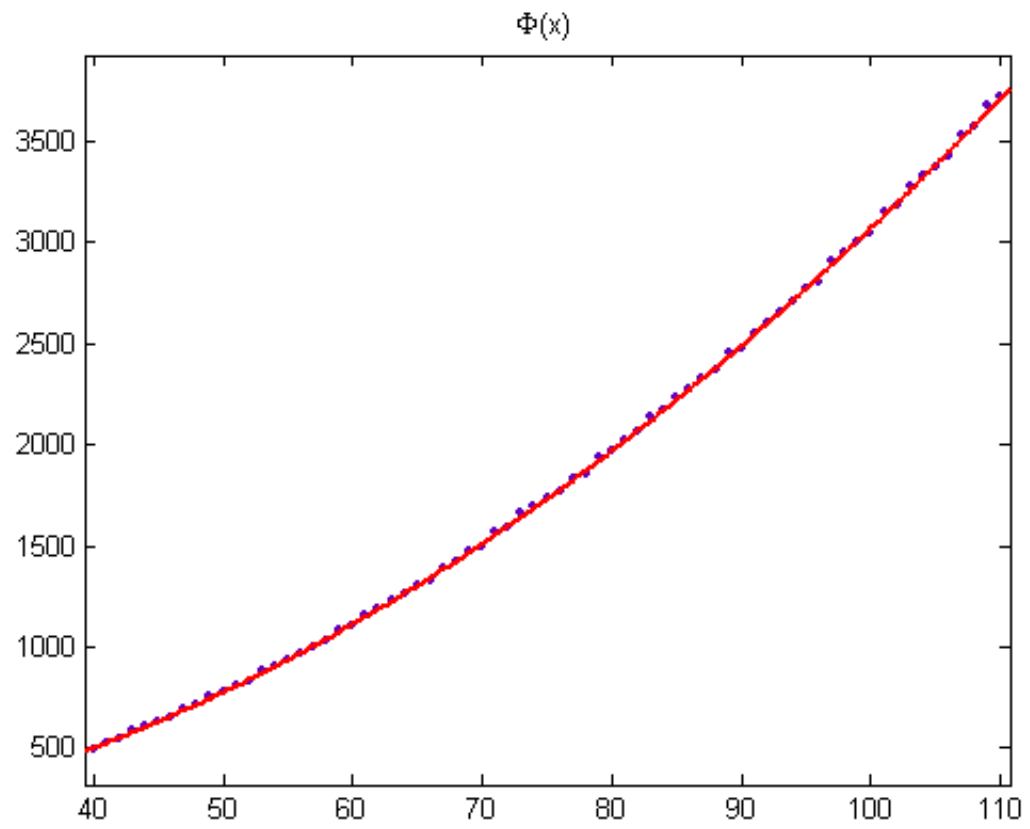

Figure 3: Plot of the $\Phi(x)$ values

For a quadratic least-squares fit of the data, $p_{1}=0.3057$ with a $95 \%$ confidence interval of $(0.2982,0.3132), p_{2}=0.0276$ with a $95 \%$ confidence interval of $(-1.103,1.158), p_{3}=10.79$ with a $95 \%$ confidence interval of $(-29.57,51.15)$, $\mathrm{SSE}=9565, \mathrm{R}$-squared $=0.9998$, and $\mathrm{RMSE}=11.86$. For a quadratic least-squares fit of the approximation, $\mathrm{SSE}=1.311 \cdot 10^{4}$, R-squared=0.9998, and RMSE=13.88. (SSE denotes sum-squared error and RMSE denotes root-mean-square error.) A plot of the relative errors is given in Figure 4. 


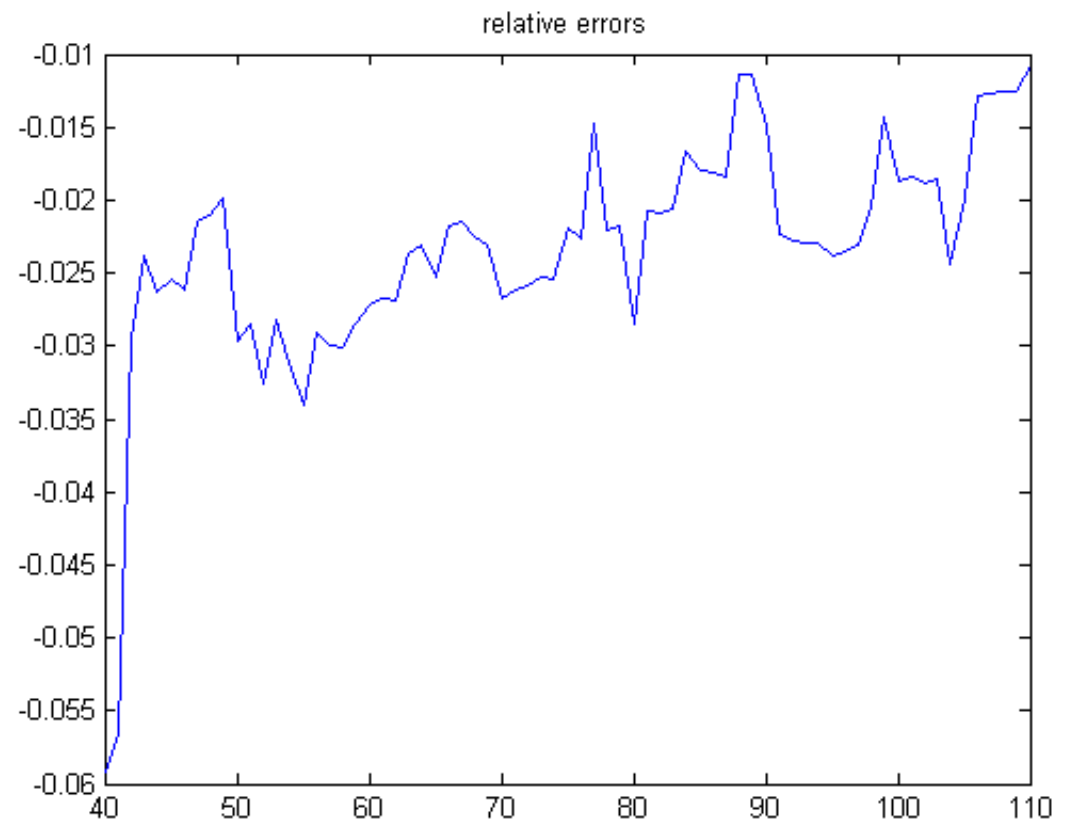

Figure 4: Plot of the relative errors

\section{The Second Chebyshev Function}

Let $U_{x}$ denote the matrix obtained from $T_{x}$ by element-by-element multiplication of the columns by $\Lambda(j)$. A plot of the sums of the columns for $x=30$ is is given in Figure 5. 


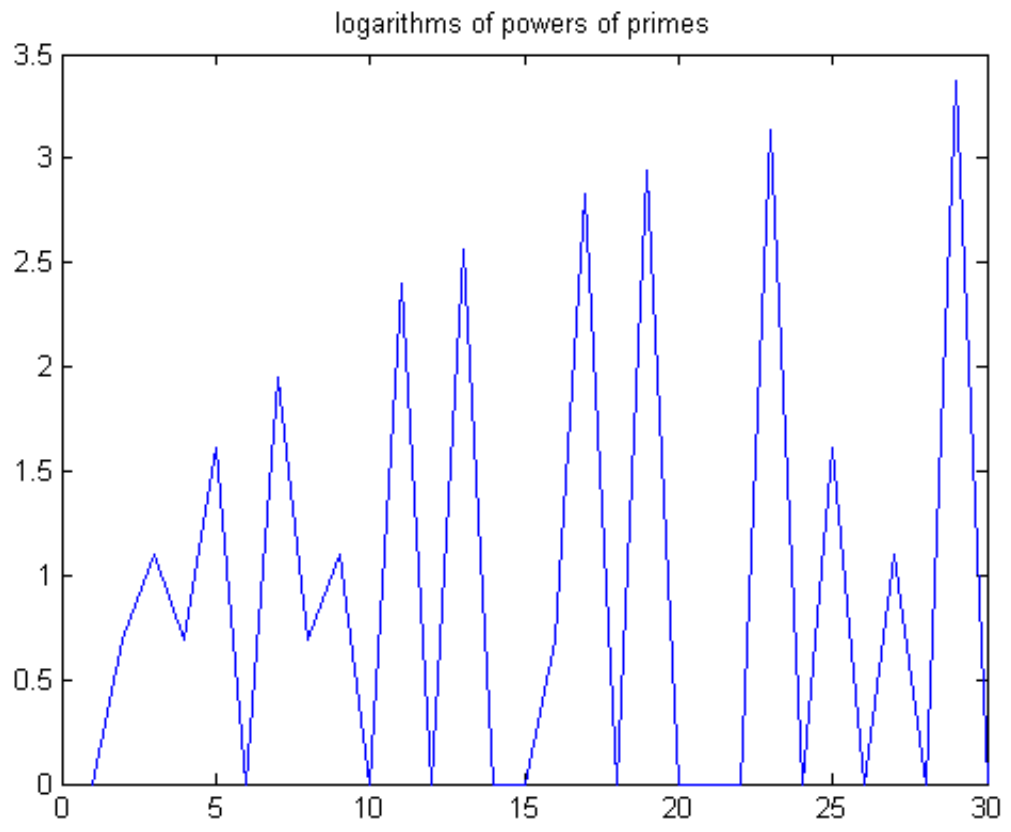

Figure 5: Plot of the sums of the columns for $x=30$

The sum of the sums of the columns is then $\psi(x)$. Let $r_{n}, n=1,2,3, \ldots, x$, denote the sums of the rows. A plot of $r_{n}$ for $n=1,2,3, \ldots 200$ is given in Figure 6. 


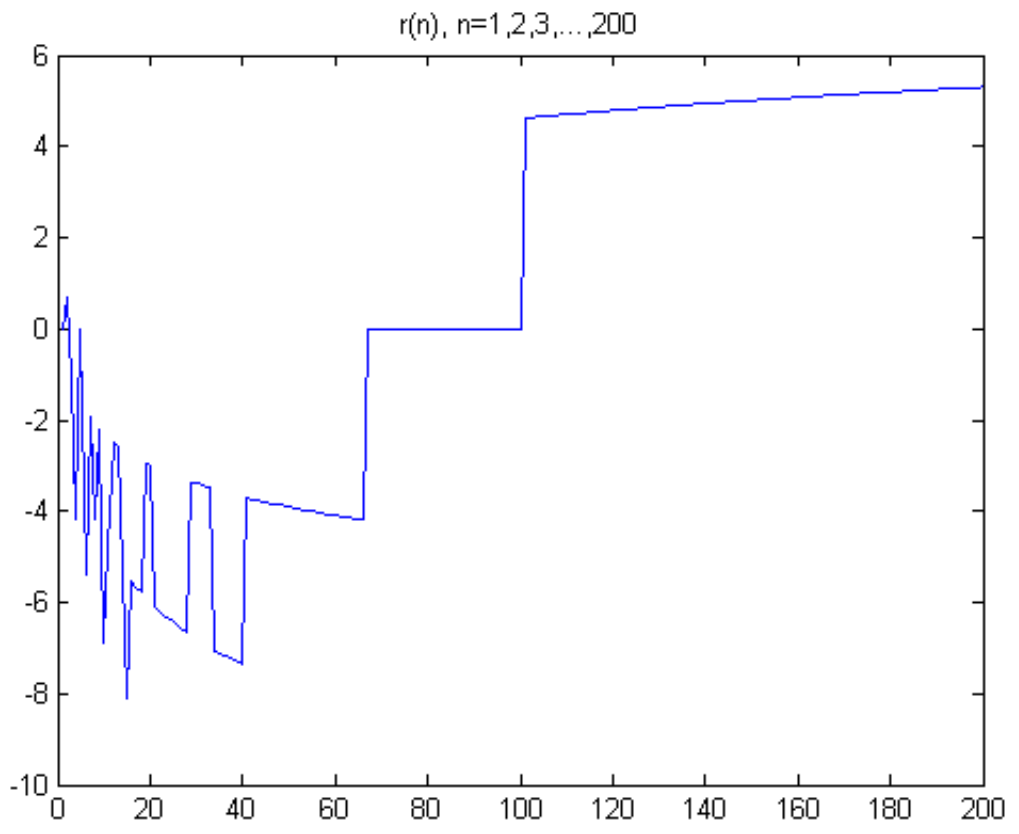

Figure 6: Plot of $r_{n}$ for $n=1,2,3, \ldots 200$

This plot indicates that $\psi(x)$ equals a series involving the squarefree numbers $1,2,3,5,6,7,10, \ldots$ Each of the "line segments" in the above plot is approximately quadratic. For $x=200$, the $r_{n}$ values from $n=\lfloor x / 2\rfloor+1$ to $\lfloor x / 1\rfloor$ (101, $102,103, \ldots, 200)$ are $\log (101), \log (102), \log (103), \ldots, \log (200)$. A plot of these values is given in Figure 7. 
$r(n)$ values, $n=101,102,103, \ldots, 200$

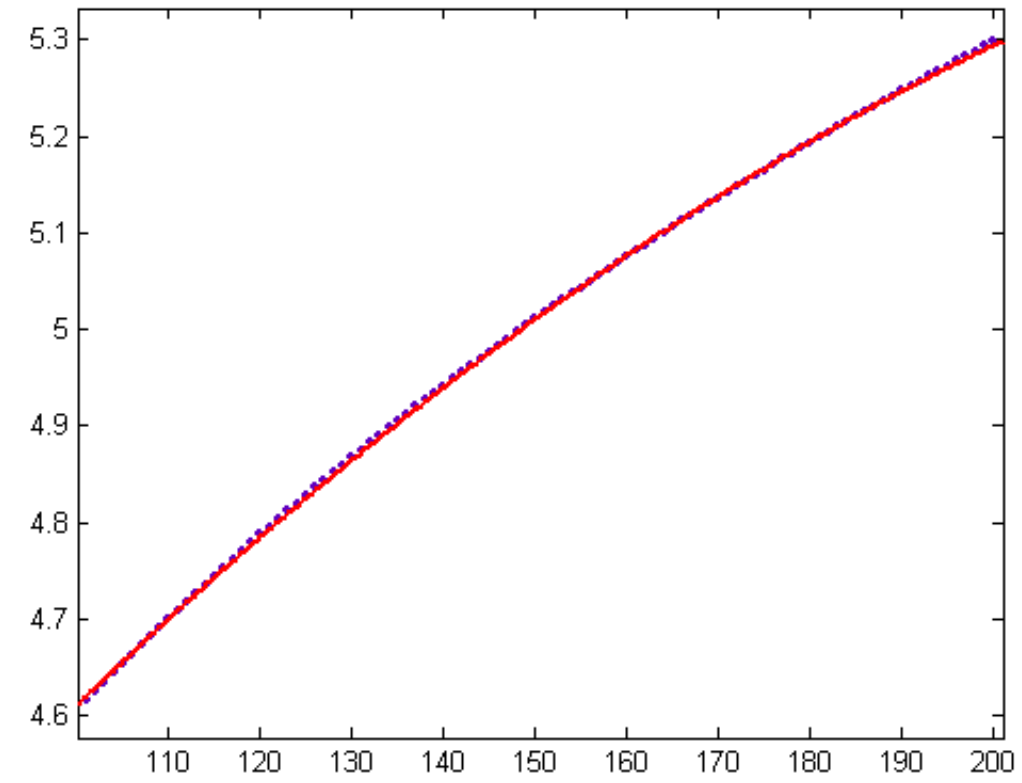

Figure 7: Plot of values from $n=\lfloor x / 2\rfloor+1$ to $\lfloor x / 1\rfloor(101,102,103, \ldots, 200)$

For a quadratic least-squares fit of the data, $p_{1}=-2.319 \cdot 10^{-5}$ with a $95 \%$ confidence interval of $\left(-2.373 \cdot 10^{-5},-2.264 \cdot 10^{-5}\right), p_{2}=0.01378$ with a $95 \%$ confidence interval of $(0.01361,0.01394), p_{3}=3.466$ with a $95 \%$ confidence interval of $(3.454,3.478), \mathrm{SSE}=0.0004042, \mathrm{R}$-squared $=0.9999$, and $\mathrm{RMSE}=0.002041$.

The $r_{n}$ values from $n=\lfloor x / 3\rfloor+1$ to $\lfloor x / 2\rfloor(67,69,70, \ldots, 100)$ are 0 . The $r_{n}$ values from $n=\lfloor x / 5\rfloor+1$ to $\lfloor x / 3\rfloor(41,42,43, \ldots, 66)$ are $-\log (41),-\log (42)$, $-\log (43), \ldots,-\log (66)$. A plot of these values is given in Figure 8 . 


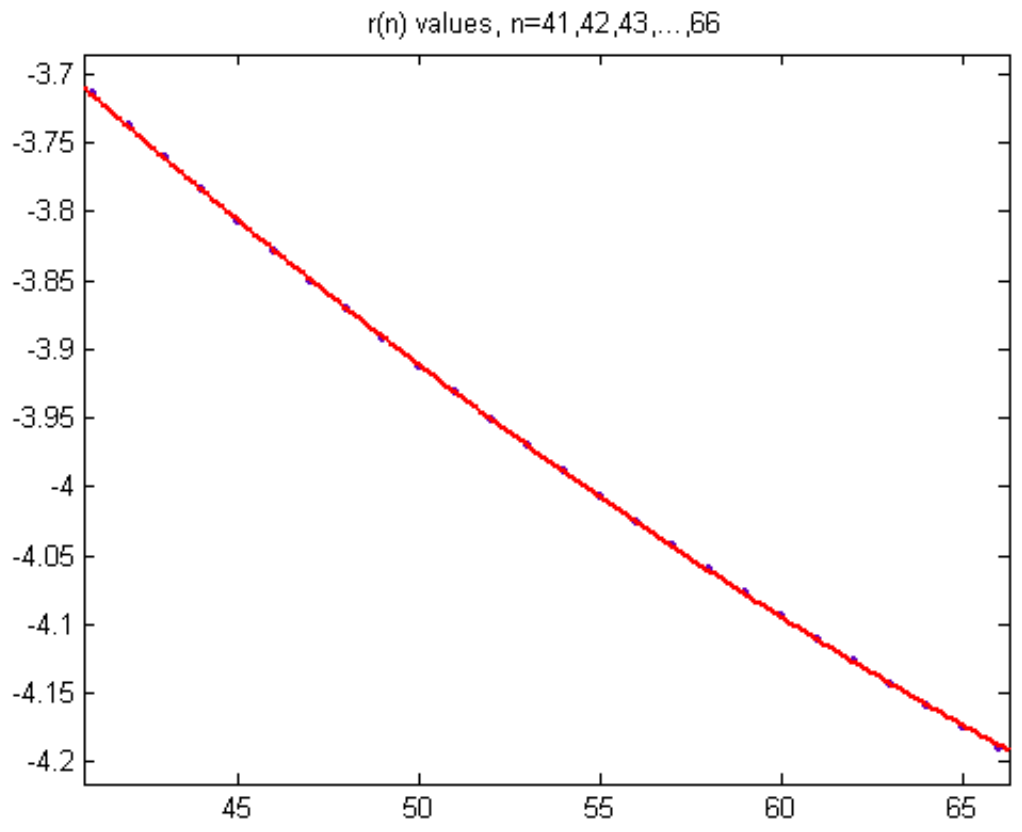

Figure 8: Plot of $r_{n}$ values from $n=\lfloor x / 5\rfloor+1$ to $\lfloor x / 3\rfloor(41,42,43, \ldots, 66)$

For a quadratic least-squares fit of the data, $p_{1}=0.0001795$ with a $95 \%$ confidence interval of $(0.0001728,0.0001857), p_{2}=-0.03809$ with a $95 \%$ confidence interval of $(-0.03878,-0.03741), p_{3}=-0.2 .455$ with a $95 \%$ confidence interval of $(-2.473,-2.437), \mathrm{SSE}=1.454 \cdot 10^{-5}, \mathrm{R}$-squared $=1$, and $\mathrm{RMSE}=0.000795$.

The $r_{n}$ values from $n=\lfloor x / 6\rfloor+1$ to $\lfloor x / 5\rfloor(34,35,36, \ldots, 40)$ are $-2 \log (34)$, $-2 \log (35),-2 \log (36), \ldots,-2 \log (40)$. A plot of these values is given in Figure 9. 


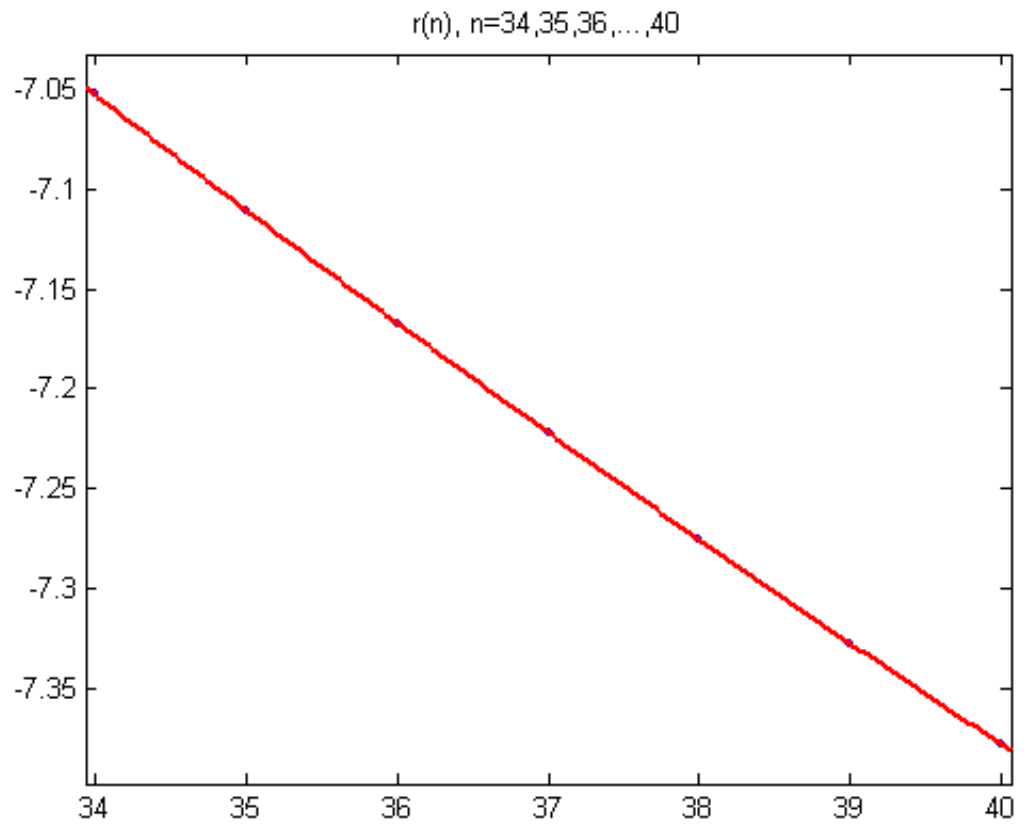

Figure 9: Plot of $r_{n}$ values from $n=\lfloor x / 6\rfloor+1$ to $\lfloor x / 5\rfloor(34,35,36, \ldots, 40)$

For a quadratic least-squares fit of the data, $p_{1}=0.000733$ with a $95 \%$ confidence interval of $(0.0007035,0.0007625), p_{2}=-0.1084$ with a $95 \%$ confidence interval of $(-0.1106,-0.1062), p_{3}=-4.215$ with a $95 \%$ confidence interval of $(-4.255,-4.175), \mathrm{SSE}=3.795 \cdot 10^{-8}, \mathrm{R}$-squared $=1$, and $\mathrm{RMSE}=9.75 \cdot 10^{-5}$.

As with $\Phi(x)$, the process continues with larger squarefree divisors. Again, the multiple for a squarefree number $q$ is $M(q-1)$. A plot of the partial sum of the Chebyshev function corresponding to the squarefree numbers $2,3,5,6,7$, and 10 is given in Figure 10. 


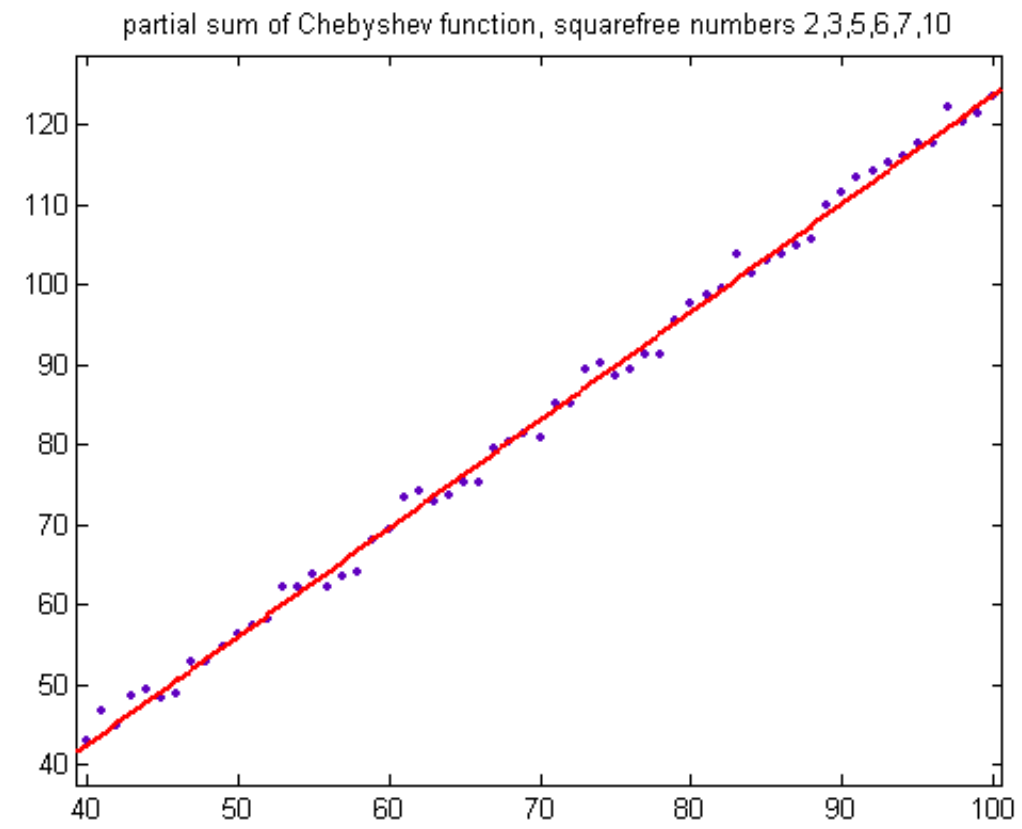

Figure 10: Plot of the partial sum of the Chebyshev function corresponding to the squarefree numbers $2,3,5,6,7$, and 10

For a linear least-squares fit of the data, $p_{1}=1.353$ with a $95 \%$ confidence interval of $(.1331,1.374), p_{2}=-11.53$ with a $95 \%$ confidence interval of $(-13.09$, $-10.02), \mathrm{SSE}=125.3, \mathrm{R}$-squared $=0.9964$, and $\mathrm{RMSE}=1.457$. The slope is greater than 1 and the SSE and RMSE are less than that for $\psi(x)$. A form of the prime number theorem is

(8) $\lim _{x \rightarrow \infty} \frac{\psi(x)}{x}=1$

See Theorem 4.4 of Apostol's book.

For $x=20, \psi(x)=19.26566$. The next $x$ value is not a prime power, so the value does not change. This is effected in the above by the logarithms of the factors of 21 canceling out $-\log (21)-\log (7)-\log (3)=0$. Similarly, the $\operatorname{logarithms}$ of the factors of 22 cancel out $-\log (22)-\log (11)-\log (2)=0$. The next $x$ value is prime. For $x=23, \psi(x)=22.40115$, an increase of $\log (23)$. The next $x$ value is not a prime power. The logarithms of the factors of 24 cancel out $-\log (24)-\log (12)-\log (8)+\log (6)+\log (4)-\log (3)-\log (2)=0$. 


\section{The Function $H(x)$}

Let $S_{x}$ denote the matrix obtained from $T_{x}$ by element-by-element multiplication of the columns by $\log (j)$. Let $r_{n}, n=1,2,3, \ldots, x$ denote the sums of the rows of $S_{x}$. A plot of $r_{n}$ for $x=500$ is given in Figure 11.

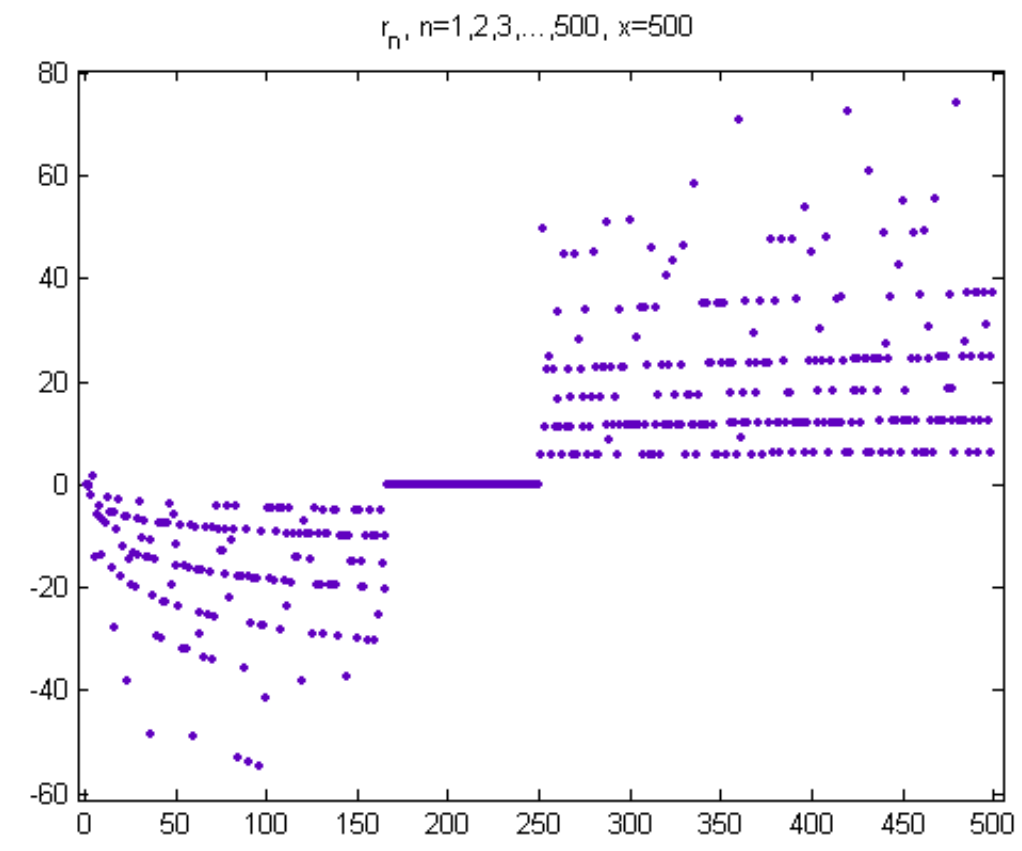

Figure 11: Plot of $r_{n}$ for $x=500$

The negative curves are derived from the logarithms of quadratic curves. A plot of the exponential of the negatives of one of the curves (the third one) is given in Figure 12. 


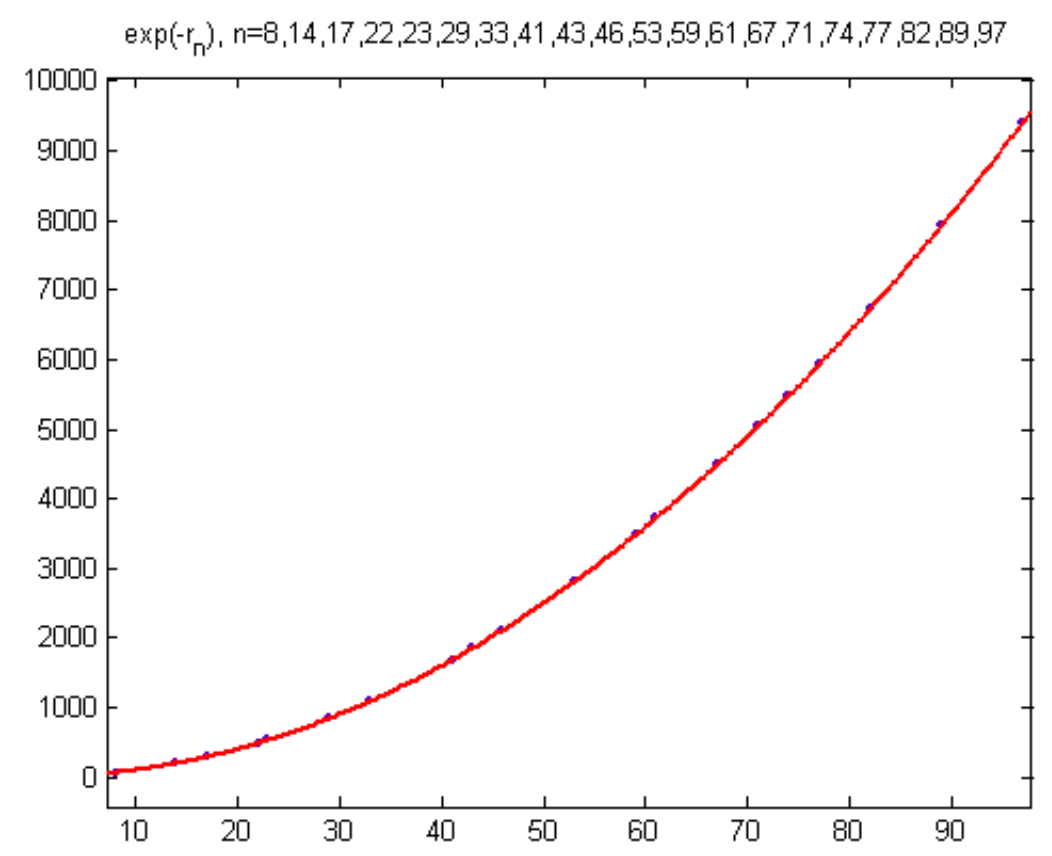

Figure 12: Plot of the exponential of the negatives of one of the curves (the third one)

For a quadratic least-squares fit of the data, $p_{1}=1$ with a $95 \%$ confidence interval of $(0.9999,1), p_{2}=0.002261$ with a $95 \%$ confidence interval of $(-0.007718$, $0.01224), p_{3}=-0.03387$ with a $95 \%$ confidence interval of $(-0.2574,0.1897)$, $\mathrm{SSE}=0.2631$, R-squared $=1$, and $\mathrm{RMSE}=0.1244$. The positive curves are also quadratic. A plot of one of the curves for $x=100$ is given in Figure 13. 
$r_{n}$ values at $n=51,55,57,58,62,65,69,74,77,82,85,86,87,91,93,94,95$

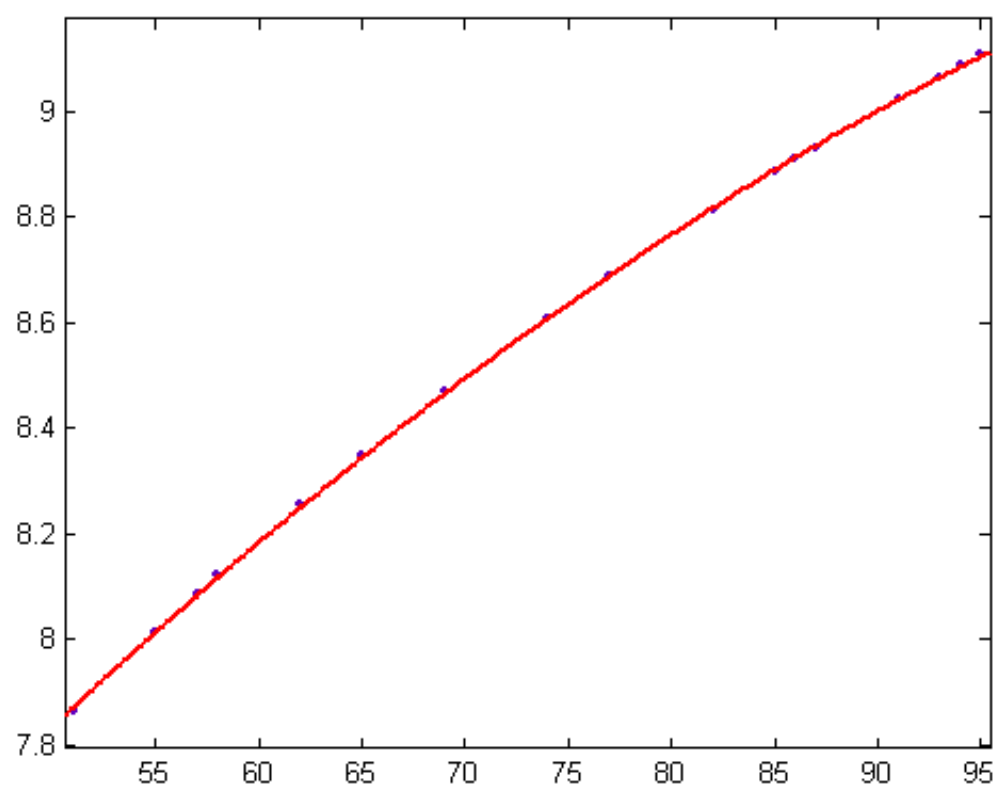

Figure 13: Plot of one of the curves for $x=100$

For a quadratic least-squares fit of the data, $p_{1}=-0.0001926$ with a $95 \%$ confidence interval of $(-0.0002054,-0.0001798), p_{2}=0.05611$ with a $95 \%$ confidence interval of $(0.05421,0.05801), p_{3}=5.511$ with a $95 \%$ confidence interval of $(5.5443,5.579), \mathrm{SSE}=0.0002127$, R-squared $=0.9999$, and $\mathrm{RMSE}=0.003898$. The number of negative or positive curves is equal to the number of distinct non-zero Mertens function values less than or equal to $x$. Sums involving step functions of certain types can be expressed as integrals by means of the following theorem.

(9) Abel's identity. For any arithmetical function $a(n)$ let $A(x)=\sum_{n \leq x} a(n)$, where $A(x)=0$ if $x<1$. Assume $f$ has a continuous derivative on the interval $[y, x]$ where $0<y<x$. Then we have $\sum_{y<n \leq x} a(n) f(n)=A(x) f(x)-$ $A(y) f(y)-\int_{y}^{x} A(t) f^{\prime}(t) d t$.

See Theorem 4.2 of Apostol's book. In the above case, Riemann-Stieltjes integration of Mertens function values is involved. The integral can be determined empirically - it turns out to be half $A(x) f(x)$. A simplified formula results since $A(1) f(1)=0$. The following theorem shows that the behavior of $M(x) / x$ is determined by that of $H(x) /(x \log x)$.

(10) We have $\lim _{x \rightarrow \infty}\left(\frac{M(x)}{x}-\frac{H(x)}{x \log x}\right)=0$. 
See Theorem 4.13 of Apostol's book. In this case, the trivial estimate $M(x)=$ $O(x)$ of $\int_{1}^{x} \frac{M(t)}{t} d t$ is used.

\section{The Selberg Identity}

Let $R_{x}$ denote the matrix obtained from $T_{x}$ by element-by-element multiplication of the columns by $\Lambda(j) \log j+\sum_{d \mid j} \Lambda(d) \Lambda\left(\frac{j}{d}\right)$. Let $r_{n}, n=1,2,3, \ldots, x$ denote the sums of the rows of $R_{x}$. A plot of $r_{n}$ for $x=500$ is given in Figure 14.

$r_{n}$ for $n=1,2,3, \ldots, 500, x=500$

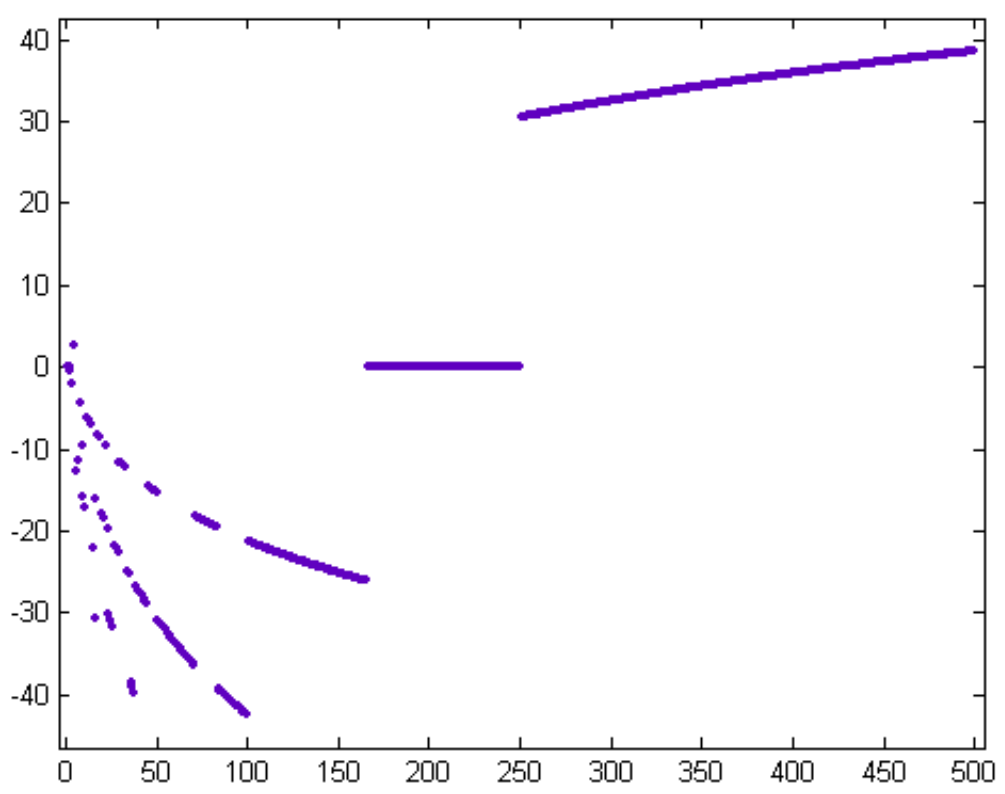

Figure 14: Plot of $r_{n}$ for $x=500$

A plot of the $r_{n}$ for $n=\lfloor x / 2\rfloor+1$ for $x=100$ is given in Figure 15 . 


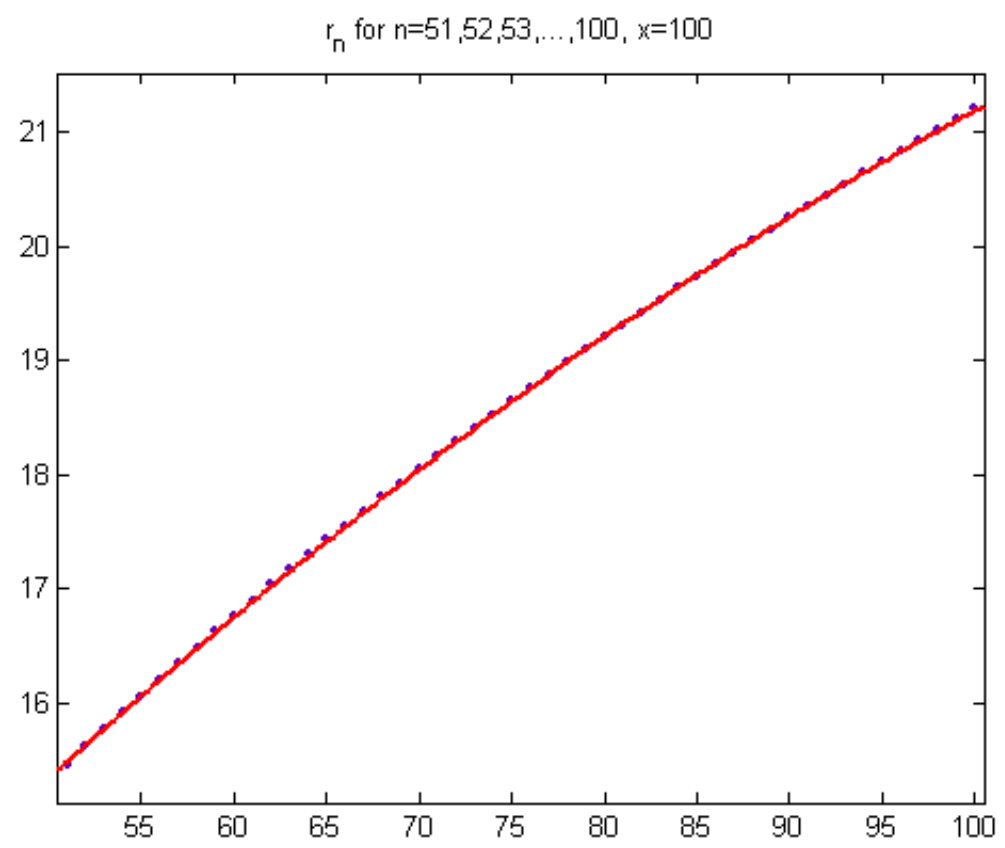

Figure 15: Plot of $r_{n}$ for $n=51,52,53, \ldots, 100, x=100$

For a quadratic least-squares fit of the data, $p_{1}=-0.0006047$ with a $95 \%$ confidence interval of $(-0.0006221,-0.0005874), p_{2}=0.2067$ with a $95 \%$ confidence interval of $(0.2049,0.2102), p_{3}=6.475$ with a $95 \%$ confidence interval of $(6.379$, 6.572), $\mathrm{SSE}=0.006063$, R-squared $=1$, and $\mathrm{RMSE}=0.01136$.

A plot of one of the negative curves is given in Figure 16. 


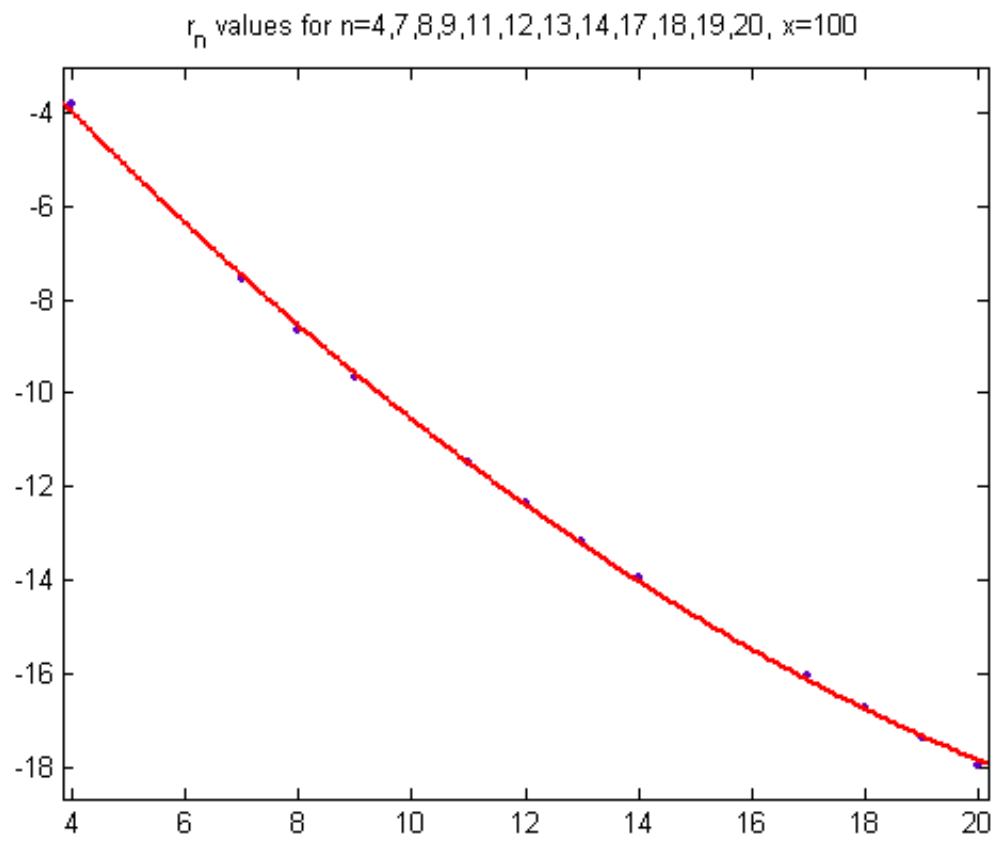

Figure 16: Plot of $r_{n}$ for $n=4,7,8,9,11,12,13,14,17,18,19$, and $20, x=100$

For a quadratic least-squares fit of the data, $p_{1}=0.02285$ with a $95 \%$ confidence interval of $(0.02011,0.02559), p_{2}=-1.415$ with a $95 \%$ confidence interval of $(-1.484,-1.345), p_{3}=1.312$ with a $95 \%$ confidence interval of $(0.9132,1.711)$, $\mathrm{SSE}=0.07941, \mathrm{R}$-squared $=0.9996$, and $\mathrm{RMSE}=0.09393$.

\section{A Product Formula for $\varphi(n)$}

The sum for $\varphi(n)$ in Theorem 2.3 of Apostol's book can also be expressed as a product extended over the distint prime divisors of $n$.

(11) For $n \geq 1$ we have $\varphi(n)=n \prod_{p \mid n}\left(1-\frac{1}{p}\right)$

See Theorem 2.4 in Apostol's book. Let $V_{x}$ denote the matrix obtained from $T_{x}$ by element-by-element multiplication of the columns by $j$. The sum of the sums of the columns is then $x(x+1) / 2$. Let $r_{n}, n=1,2,3, \ldots, x$, denote the sums of the rows. A plot of $r_{n}$ for $x=200$ is given in Figure 17 . 
$r(n)$ for $n=1,2,3, \ldots, 200, x=200$

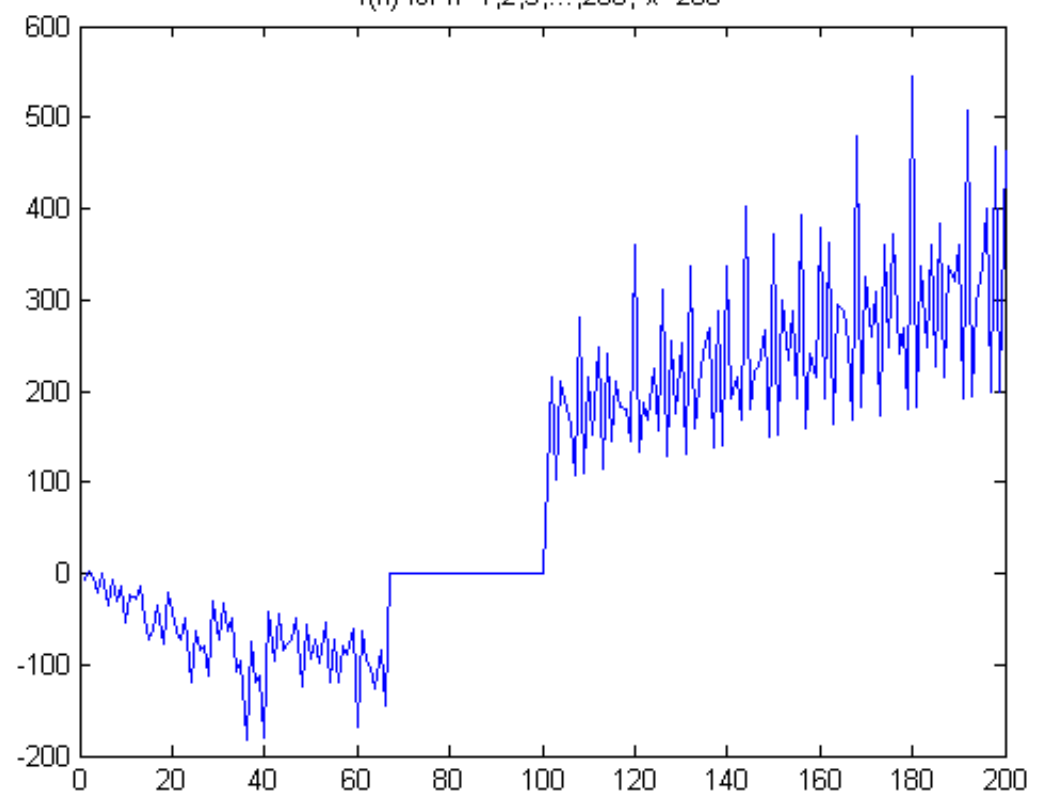

Figure 17: Plot of $r_{n}$ for $x=200$

The same intervals corresponding to the squarefree numbers occur and the same multiples of the Mertens function are applicable. In the following, the $r_{n}$ values are "normalized", that is, divided by the corresponding Mertens function value (except when that value is 0 ). Let $r_{n}^{\prime}$ denote the normalized values. $r_{n}$ values of zero are neglected. A product formula similar to Euler's is applicable, but $p+1$ factors occur instead of $p-1$ factors.

(12) If $n$ is squarefree, $\prod_{p \mid n}(p+1)=r_{n}^{\prime}$.

When $n$ is not squarefree, more complex relationships occur. For example,

(13) If $n=p_{1}^{2} p_{2}, r_{n}^{\prime}=\left(p_{2}+1\right)\left(p_{1}\left(p_{1}+1\right)+1\right)$.

Let $\sigma(n)$ denote the sum of positive divisors function. Let $W_{x}$ denote the matrix obtained from $T_{x}$ by element-by-element multiplication of the columns by $\sigma(j)$. Let $r_{n}, n=1,2,3, \ldots, x$, denote the sums of the rows. A plot of $r_{n}$ for $x=200$ is given in Figure 18. 
$r(n), n=1,2,3, \ldots, 200, x=200$

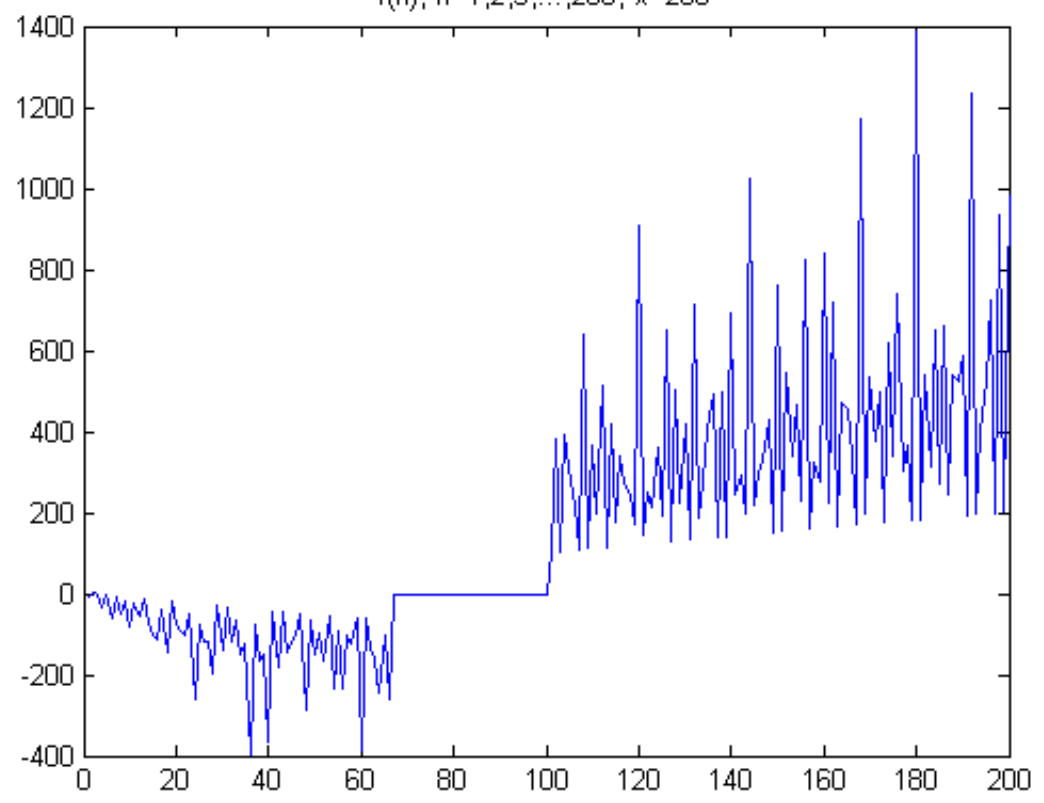

Figure 18: Plot of $r_{n}$ for $x=200$

The same intervals corresponding to the squarefree numbers occur and the same multiples of the Mertens function are applicable. In the following, the $r_{n}$ values are "normalized". A product formula similar to Euler's is applicable, but $p+2$ factors occur instead of $p-1$ factors.

(14) If $n$ is squarefree, $\prod_{p \mid n}(p+2)=r_{n}^{\prime}$.

If $n$ is not squarefree, more complex relationships occur. For example,

(15) If $n=p_{1}^{2} p_{2}$ and $p_{1}>3, r_{n}^{\prime}=\left(p_{2}+2\right)\left(p_{1}\left(p_{1}+2\right)+3\right)$.

\section{An Analogue of $\Phi(x)$}

Let $Y_{x}$ denote the matrix obtained from $T_{x}$ by element-by-element multiplication of the columns by $\sigma(j)-j$. Let $c_{n}, n=1,2,3, \ldots, x$ denote the sums of the columns of $Y_{x}$. A plot of $c_{n}$ for $x=100$ is given in Figure 19 . 


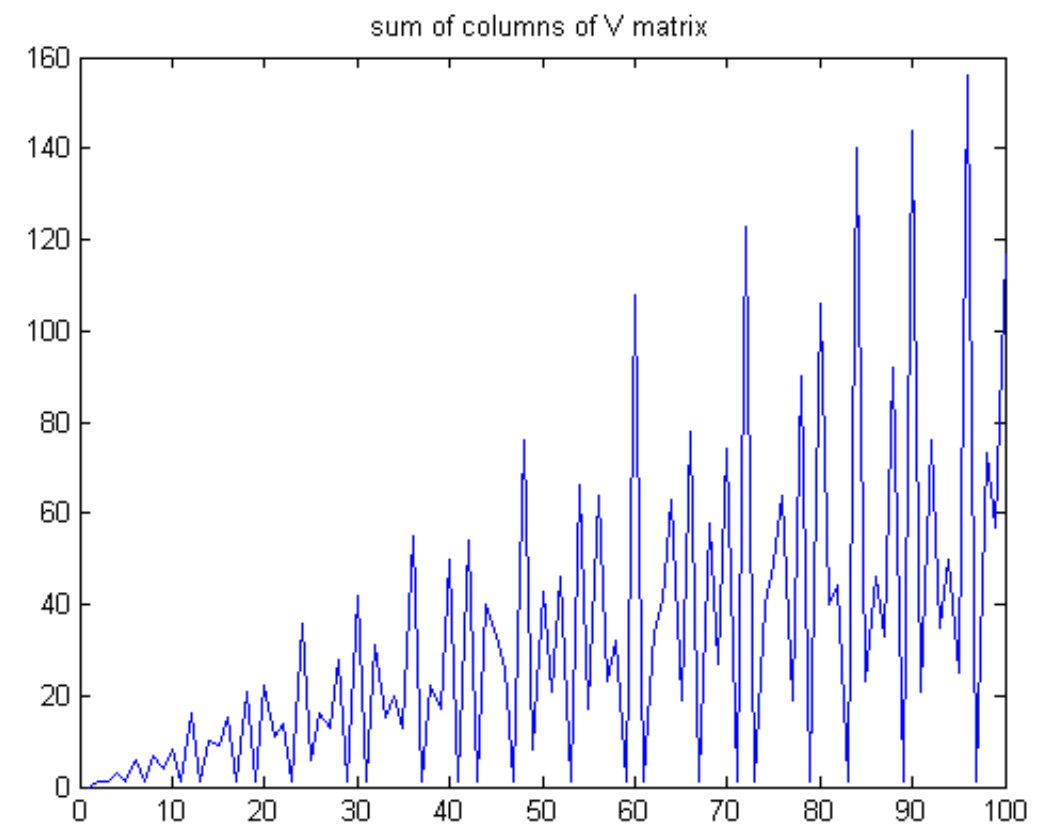

Figure 19: Plot of $c_{n}$ for $x=100$

$\sum_{n=1}^{x} c_{n}$ is approximately equal to $\Phi(x)$. For $x=100$, the sum of the sums of the columns is 3249 whereas $\Phi(x)=3044$. An empirical result is

(16) $c_{n}$ where $n>1$ is positive. $c_{1}=0$.

Let $r_{n}, n=1,2,3, \ldots, x$ denote the sums of the rows of $Y_{x}$. A plot of $r_{n}$ for $x=100$ is given in Figure 20. 


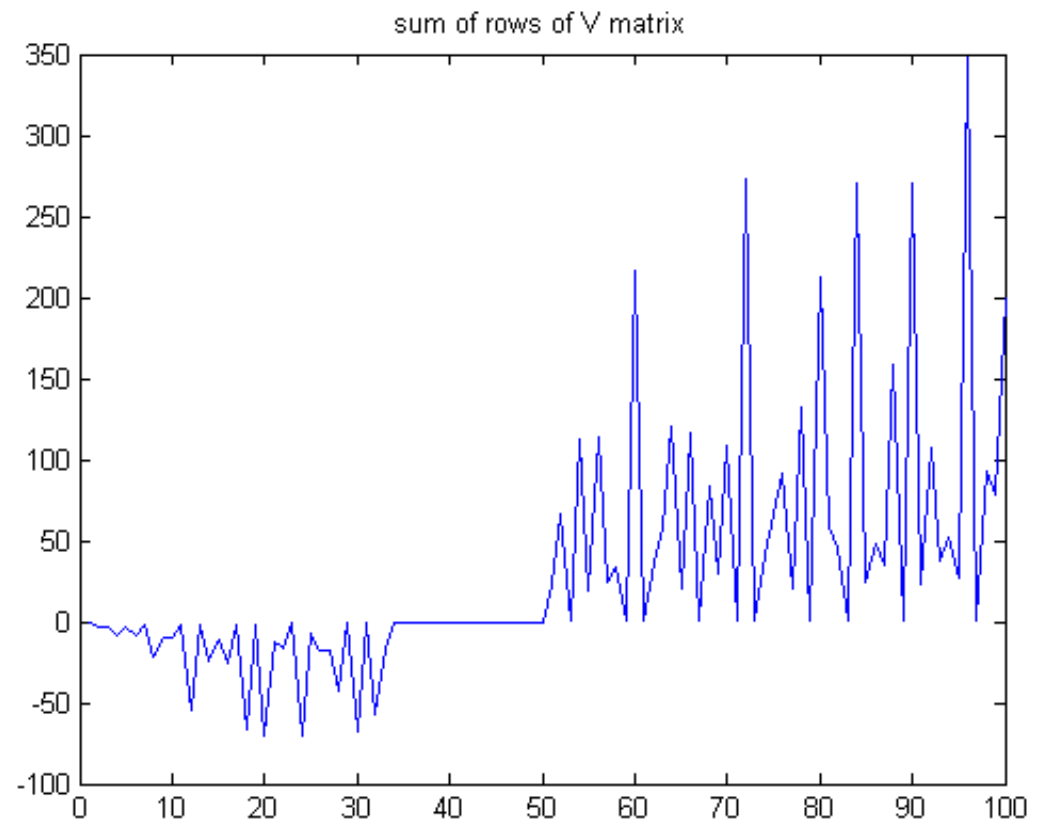

Figure 20: Plot of $r_{n}$ for $x=100$

Compared to the sums of the rows of $U_{x}$, the sums of the rows of $Y_{x}$ have been "normalized". An empirical result is

(17) $r_{n}$ is usually non-positive for $n<\lfloor x / 3\rfloor+1$, positive for $n>\lfloor x / 2\rfloor$, and 0 in between.

A plot of the upper bounds of the $n$ values, $n<\lfloor x / 3\rfloor+1$, where $r_{n}$ becomes greater than 0 for $x=200,300,400, \ldots, 11100$ is given in Figure 21 . 


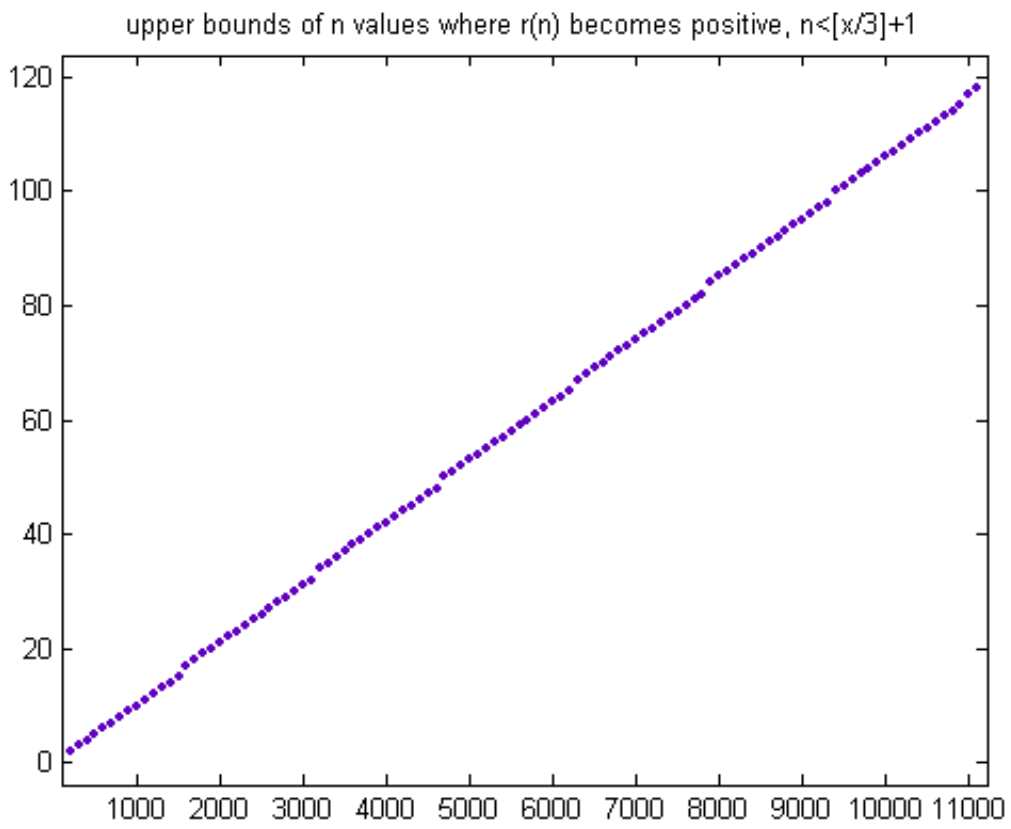

Figure 21: Plot of the upper bounds of the $n$ values, $n<\lfloor x / 3\rfloor+1$

The plot is not linear - the values jump by 2 at $x=1600,3200,4800,6200$, 7800,9400 , and 11000 . Otherwise, the values increase by 1 at every $x$ multiple of 100. For example, for $x=1601,1602$, and 1603 , the $\left(n, r_{n}\right)$ values where $r_{n}>0$ are $(2,1),(4,4),(7,3),(16,26)$, and $(17,1)$. For $x=1604,1605$, and 1606 , the $\left(n, r_{n}\right)$ values are $(2,2),(7,2),(16,26)$, and $(17,1)$. For $x=1607$, 1608 , and 1609 the $\left(n, r_{n}\right)$ values are $(2,3),(7,2),(11,1),(16,26)$, and $(17$, 1 ). As $x$ increases, the number of positive $r_{n}$ values less than the upper bound increases but in an erratic fashion. Note that the $r_{n}$ values at prime $n$ values are small if not equal to 1 .

\section{Additive Properties of $r_{n}$ (for $Y_{x}$ )}

An empirical result is

(18) $r_{n}=1$ if and only if $n$ is prime. If $r_{n}=-1$, then $n$ is prime.

Let $p, q, r$, and $s$ denote distinct primes. An empirical result is

(19) When $1<n<\lfloor x / 3\rfloor+1, r_{n}$ values at $n=p^{2}$ equal $-(p+2)$, small positive multiples of $-(p+2)$, or 0 . For $n>\lfloor x / 2\rfloor$, the $r_{n}$ values at $n=p^{2}$ equal $p+2$. 
There do not appear to be analogous properties for $r_{n}$ values at $n=p^{3}, n=p^{4}$, $n=p^{5}$, etc.

(20) When $1<n<\lfloor x / 3\rfloor+1, r_{n}$ values at $n=p q$ equal $-(p+q+3)$, small positive multiples of $-(p+q+3)$, or 0 . For $n>\lfloor x / 2\rfloor$, the $r_{n}$ values at $n=p q$ equal $p+q+3$.

(21) $\left|r_{n}\right|$ values at $n=p q r$ equal either 0 or rational multiples of $(p+q)+(p+$ $r)+(q+r)$ minus a natural number. For $p=2, q=3$, and $r=5,7,11, \ldots$, the multiple is 4 and the natural number is 12 . For $p=2, q=5$, and $r=7,11$, $13, \ldots$, the multiple is 5 and the natural is 32 . For $p=2, q=7$, and $r=11,13$, $17, \ldots$, the multiple is 6 and the natural number is 60 . For $p=2, q=11$, and $r=13,17,19, \ldots$, the multiple is 8 and the natural number is 140 . In general, the multiple is $(q+5) / 2$. For $p=3, q=5$, and $r=7,11,13, \ldots$, the multiple is $\frac{11}{2}$ and the natural number is 42 .

(22) $\left|r_{n}\right|$ values at $n=p q r s$ equal either 0 or rational multiples of $(p+q)+(p+$ $r)+(p+s)+(q+r)+(q+s)+(r+s)$ minus a natural number. For $p=2$, $q=3, r=5$, and $s=7,11,13, \ldots$, the multiple is $\frac{68}{3}$ and the natural number is 472 .

(23) When $1<n<\lfloor x / 3\rfloor+1, r_{n}$ values at $n=p^{2} q$ equal $-(m(p+q)+i)$, small positive multiples of $-(m(p+q)+i)$, or 0 where $m$ and $i$ are natural numbers. For $n>\lfloor x / 2\rfloor$, the $r_{n}$ values at $n=p^{2} q$ equal $m(p+q)+i$. For $p=2$ and $q=3,5,7, \ldots, m=4$ and $i=7$. For $p=3$ and $q=5,7,11, \ldots, m=5$ and $i=8$.

These and similar relationships appear to account for all $n$ values.

The first few superabundant numbers $(\sigma(n) / n>\sigma(k) / k$ for $1 \leq k \leq n-1)$ are $2,6,12,60,120,360,2520$, and 5040. The prime factorizations are $2,2 \cdot 3,2^{2} \cdot 3$, $2^{2} \cdot 3 \cdot 5,2^{3} \cdot 3 \cdot 5,2^{3} \cdot 3^{2} \cdot 5,2^{3} \cdot 3^{2} \cdot 5 \cdot 7$, and $2^{4} \cdot 3^{2} \cdot 5 \cdot 7$ and the $\sigma(n) / n$ values are $1.5,2.0,2.333,2.8,3.0,3.25,3.714,3.838$. Similar superabundant numbers can be found for a fixed $x$ value - the largest $\sigma\left(\left|r_{n}\right|\right) /\left|r_{n}\right|, n=1,2,3, \ldots, x$ value is found. For $x=2,6,12,60,120,360,2520$, and 5040, the "superabundant numbers" are $2,8,10,84,180,-240,7560$, and 32760 . The prime factorizations are $2,2^{3}, 2 \cdot 5,2^{2} \cdot 3 \cdot 7,2^{2} \cdot 3^{2} \cdot 5,2^{4} \cdot 3 \cdot 5,2^{3} \cdot 3^{3} \cdot 5 \cdot 7$, and $2^{3} \cdot 3^{2} \cdot 5 \cdot 7 \cdot 13$ and the $\sigma\left(\left|r_{n}\right|\right) /\left|r_{n}\right|$ values are $1.0,1.875,1.8,2.667,3.033,3.1,3.809$, and 4.0 .

\section{Ramanujan and Robin's Theorems}

Let $\gamma$ denote Euler's constant.

(24) $\lim \sup _{n \rightarrow \infty} \frac{\sigma(n)}{n \log \log n}=e^{\gamma}$ 
(25) $\liminf _{n \rightarrow \infty} \frac{\varphi(n) \log \log n}{n}=e^{-\gamma}$

See 18.3 of Hardy and Wright's [5] book. Ramanujan [6] proved that the Riemann hypothesis implies $\sigma(n)<e^{\gamma} n \log \log n$ for sufficiently large $n$. Robin [7] proved that this inequality is true for all $n \geq 5041$ if and only if the Riemann hypothesis is true. Robin's theorems are

(26) If the Riemann hypothesis is true, then for each $n \geq 5041, \sum_{d \mid n} d \leq$ $e^{\gamma} n \log \log n$ where $\gamma$ is Euler's constant.

(27) If the Riemann hypothesis is false, then there exist constant $0<\beta<\frac{1}{2}$ and $C>0$ such that $\sum_{d \mid n} e^{\gamma} n \log \log n+\frac{C n \log \log n}{(\log n)^{\beta}}$ holds for infinitely many $n$.

The constant $\beta$ can be chosen to take any value $1-b<\beta<\frac{1}{2}$ where $b=\Re(\rho)$ for some zero $\rho$ of $\xi(s)$ with $R(\rho)>\frac{1}{2}$ and $C>0$ must be chosen sufficiently small, depending on $\rho$.

A relationship proved by Cox is

(28) $\sum_{n=1}^{x} M(\lfloor x / n\rfloor) \sigma(n)=x(x+1) / 2$

An empirical result is

$(29) e^{\gamma} \Phi(x)>x(x+1) / 2$

A plot of $e^{\gamma} \Phi(x)$ and $x(x+1) / 2$ for $x=2,3,4, \ldots, 20$ is given in Figure 22 . 


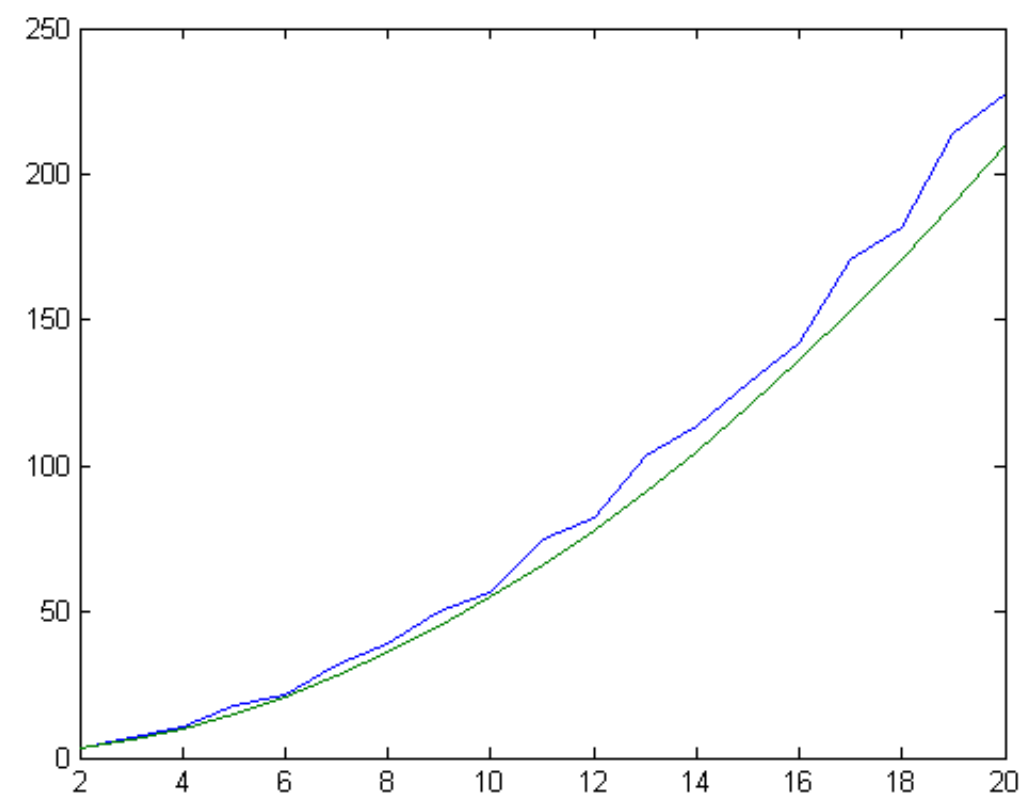

Figure 22: Plot of $e^{\gamma} \Phi(x)$ and $x(x+1) / 2$ for $x=2,3,4, \ldots, 20$

$i>\varphi(i)$ so $\frac{x(x+1)}{2}>\Phi(x)$. Upper and lower bounds of $\Phi(x)$ are then determined. These conditions may be equivalent to the Riemann hypothesis.

\section{Conclusion}

The modified Redheffer matrix technique is applicable to any formula containing the Möbius function. The Möbius function arises in many different places in number theory. The above is the basis for a more rigorous investigation (currently everything is empirically derived). Abel's identity may result in a proof.

\section{References}

[1] T. M. Apostol, Introduction to Analytic Number Theory, Springer, (1976)

[2] M. Mikolás, Farey series and their connection with the prime number problem 1, Acta Sci. Math. (Szeged) 13 (1949), 93-117

[3] R. M. Redheffer, Eine explizit lösbare Optimierungsaufgabe, Internat. Schriftenreine Numer. Math., 36 (1977) 
[4] Cox, Darrell and Ghosh, Sourangshu and Sultanow, Eldar. (2021). Bounds of the Mertens Functions. Advances in Dynamical Systems and Applications. 16. 35-44

[5] G. H. Hardy and E. M. Wright, An Introduction to the Theory of Numbers, Oxford at the Clarendon Press, (1960)

[6] Ramanujan, Srinivasa (1997), Highly composite numbers, annotated by Jean-Louis Nicolas and Guy Robin, The Ramanujan Journal, 1:(2), 119153

[7] Robin, Guy (1984), Grandes valeurs de la fonction somme des diviseurs des diviseurs et hypothèse de Riemann, Journal de Mathématiques Pures et Appliqueés, Neuvième Série 63(2); 187-213 